\title{
Long non-coding RNA H19 induces hippocampal neuronal apoptosis via Wnt signaling in a streptozotocin-induced rat model of diabetes mellitus
}

\author{
Yu-Hao Zhao ${ }^{1}$, Tie-Feng $\mathrm{Ji}^{2}$, Qi Luo ${ }^{1}$ and Jin-Lu Yu ${ }^{1}$ \\ ${ }^{1}$ Department of Neurosurgery, The First Hospital of Jilin University, Changchun, P.R. China \\ 2 Department of Radiology, The First Hospital of Jilin University, Changchun, P.R. China \\ Correspondence to: Jin-LU YU, email: jinluyu@hotmail.com \\ Qi Luo, email: liluoqi@126.com
}

Keywords: long non-coding RNA H19, hippocampal neurons, diabetes mellitus, Wnt signaling pathway, streptozotocin, Pathology Section

Received: November 24, $2016 \quad$ Accepted: March 16, $2017 \quad$ Published: April 27, 2017

Copyright: Zhao et al. This is an open-access article distributed under the terms of the Creative Commons Attribution License 3.0 (CC BY 3.0), which permits unrestricted use, distribution, and reproduction in any medium, provided the original author and source are credited.

\section{ABSTRACT}

Defects in hippocampal synaptic plasticity and disorders of memory and learning are the central nervous system complications of diabetes mellitus (DM). Here, we used a streptozotocin-induced rat DM model to investigate the effects of long noncoding RNA H19 (IncRNA H19) on learning and memory and apoptosis of hippocampal neurons, and the involvement of the Wnt signaling. Our data demonstrate that IncRNA H19 is highly expressed in rats with DM. Over-expression of IncRNA H19 increased positioning navigation latency in DM rats and decreased duration of space exploration. IncRNA H19 over-expression also increased hippocampal neuronal apoptosis and expression of Wnt3, $\beta$-catenin, TCF-1, Bax, caspase-8 and caspase-3. By contrast, expression of GSK-3 $\beta$ and $\mathrm{Bcl}-2$ was suppressed in DM rats over-expressing IncRNA H19. These results suggest that IncRNA H19 induces hippocampal neuronal apoptosis via Wnt signaling, and that inhibition of IncRNA H19 may serve as a promising novel target for the treatment of cognitive decline in patients with DM.

\section{INTRODUCTION}

Diabetes mellitus (DM) is a group of metabolic diseases characterized by hyperglycemia, insulin resistance, and/or insulin deficiency [1]. The chronic hyperglycemia of DM is associated with long-term dysfunction, damage, and multiple organ failure, particularly kidney, eye, heart, nerve, and blood vessel dysfunction [2]. In addition, DM adversely affects the brain, which undergoes changes that may increase the risk of cognitive decline [3]. Animal models with DM exhibit biochemical and behavioral deficits, particularly in the hippocampus, a brain region important for memory and learning [4]. For example, DM rats spend less time exploring novel objects, supporting a deficit of memory processing [5]. In addition, DM rats have decreased locomotor activity and higher immobility during a forced swim test [5].
Long non-coding RNAs (lncRNAs) are essential for higher cognitive abilities and brain development, and are involved in psychiatric diseases, providing tissue- and activity-specific epigenetic and transcriptional regulation [6]. $H 19$ gene is a paternally imprinted gene located near the telomeric region of chromosome $11 \mathrm{p} 15.5$, an area frequently involved in adult and pediatric tumors [7]. Almost 98\% of the "junk" DNA is transcribed to noncoding RNAs (ncRNAs), including short ncRNAs and lncRNAs [8]. The human $H 19$ gene encodes a $2.3 \mathrm{~kb}$ long, polyadenylated and spliced ncRNA that is vital in embryonal growth control [9]. The lncRNA H19 has been associated with the regulation of human tumor growth [10] and activation of the Wnt signaling pathway [11]. In addition, Wnt signaling is an essential regulator of adult hippocampal neurogenesis, and Wnt protein regulates adult hippocampal function [12]. A dynamic gradient of Wnt signaling pathway can regulate cellular specification 
in the hippocampus and initiation of neurogenesis in the mammalian cortex [13]. The modules transducing Wnt signaling are well conserved throughout evolution, and $\beta$-catenin plays a vital role in the canonical Wnt signaling [14].

In this study, we hypothesized that IncRNA H19 might regulate apoptosis of hippocampal neurons via the Wnt signaling. Using a rat DM model, we investigated the impact of lncRNA H19 on learning and memory, apoptosis of hippocampal neurons, and involvement of the Wnt signaling.

\section{RESULTS}

\section{Expression of IncRNA H19 in the hippocampal tissues}

The expression of lncRNA H19 in the hippocampus tissues was analyzed by qRT-PCR. Compared to the normal group, the expression of lncRNA H19 was significantly increased in the empty vector model, NC, and over-expressed lncRNA H19 groups (all $P<0.05$ ), while it was decreased in the lncRNA H19-shRNA group $(P<$ $0.05)$. There was no significant difference between the lncRNA H19-shRNA and normal groups $(P>0.05)$. The expression of lncRNA H19 in the over-expressed lncRNA H19 group was higher than in the model and empty vector groups (all $P<0.05$ ), and there was no statistically significant difference between these two groups $(P>$ 0.05). The expression of lncRNA H19 in the lncRNA H19-shRNA group was decreased compared to the model and NC groups $(P<0.05)$, and there was no statistically significant difference between these two groups $(P>0.05)$ (Figure 1).

\section{Whole gene methylation in rat models of the normal and model groups}

Compared to the normal group, hippocampal tissues of DM rats showed hypomethylation in the model group (Figure 2) $(P<0.01)$. In the model group, the methylation in $\mathrm{H} 19$ promoter region was lower than in the normal and NC groups (all $P<0.01$ ).

\section{Blood glucose levels and body weight in DM rats}

In the normal group, there was no significant difference in basal blood glucose levels, blood glucose levels after rat modeling, and random blood glucose levels at 12 weeks $(P>0.05)$. The basal blood glucose levels in the individual groups were not significantly different (all $P>0.05$ ). Compared to the normal group, the blood glucose level after modeling in each group significantly increased (all $P<0.05$ ). At 12 weeks, the blood glucose

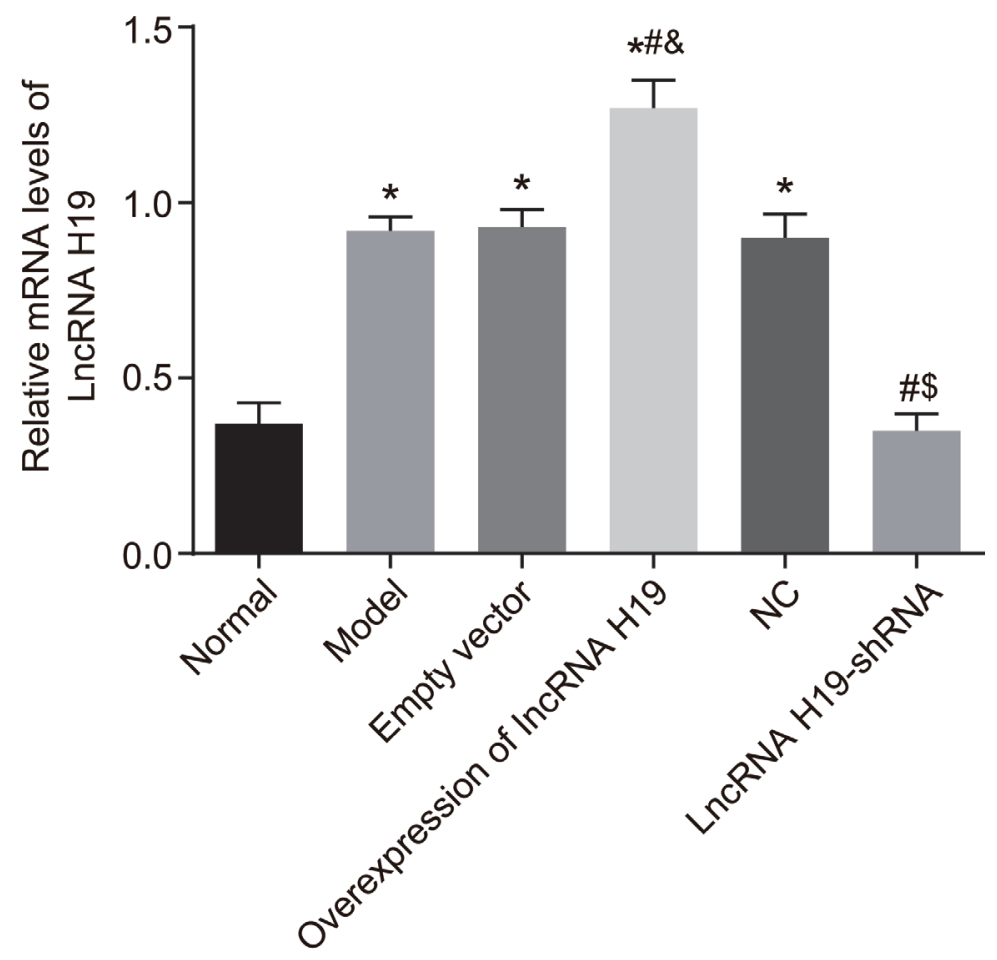

Figure 1: Expressions of Lnc RNA H19 in hippocampal tissues of rats in each group measured by qRT-PCR. Notes: $*, P<0.05$ as compared with the normal group; \#, $P<0.05$ as compared with the model group; $\&, P<0.05$ as compared with the empty vector group; $\$, P<0.05$ as compared to the $\mathrm{NC}$ group; $\mathrm{NC}=$ negative control; qRT-PCR $=$ quantitative real-time plymerace chain reaction. 
Table 1: Comparison of blood glucose $(\mathrm{mmol} / \mathrm{L})$ before and after the rat modeling in esch group $(n=10, \mathrm{mmol} / \mathrm{L})$.

\begin{tabular}{|l|c|c|c|}
\hline Group & Basal blood glucose & Blood glucose after modeling & $\begin{array}{l}\text { Random blood glucose at 12 } \\
\text { weeks }\end{array}$ \\
\hline Normal group & $6.00 \pm 0.89$ & $5.94 \pm 0.92$ & $6.29 \pm 0.85$ \\
\hline Model group & $6.02 \pm 0.85$ & $21.99 \pm 3.66^{*_{\mathrm{a}}}$ & $25.21 \pm 3.24^{* \mathrm{ab}}$ \\
\hline Empty vector group & $5.84 \pm 0.78$ & $22.27 \pm 3.52^{*_{\mathrm{a}}}$ & $25.46 \pm 3.31^{* \mathrm{ab}}$ \\
\hline Over-expressed lncRNA H19 group & $6.00 \pm 0.83$ & $22.51 \pm 3.67^{*_{\mathrm{a}}}$ & $28.79 \pm 3.22^{* \# \& \mathrm{ab}}$ \\
\hline NC group & $6.17 \pm 0.91$ & $21.69 \pm 3.51^{* \mathrm{a}}$ & $25.15 \pm 3.46^{* \mathrm{ab}}$ \\
\hline LncRNA H19-shRNA group & $6.15 \pm 0.84$ & $22.22 \pm 3.68^{* \mathrm{a}}$ & $6.31 \pm 0.91^{\# \text { ab }}$ \\
\hline
\end{tabular}

Notes: *, $P<0.05$ as compared with the normal group; \#, $P<0.05$ as compared with the model group; $\&, P<0.05$ as compared with the empty vector group; $\$, P<0.05$ as compared to the NC group; a, $P<0.05$ as compared with the basal blood glucose; $\mathrm{b}, P<0.05$ as compared with the blood glucose after the modeling; $\mathrm{NC}=$ negative control; lncRNA, long non-coding RNA; NC, negative group; shRNA, short hairpin RNA.

Table 2: Body weight (g) before and after the modeling in each group (mean $\pm s, n=10)$.

\begin{tabular}{|l|c|c|c|}
\hline Group & Basal body weight & Body weight after the modeling & Body weight at 12 weeks \\
\hline Normal group & $204.5 \pm 5.3$ & $207.4 \pm 4.8$ & $506.7 \pm 13.8$ \\
\hline Model group & $209.0 \pm 4.9$ & $214.1 \pm 4.1$ & $473.5 \pm 6.7^{* a b}$ \\
\hline Empty vector group & $210.8 \pm 3.7$ & $206.9 \pm 2.1$ & $475.2 \pm 5.4^{* a b}$ \\
\hline $\begin{array}{l}\text { Over-expressed lncRNA H19 } \\
\text { group }\end{array}$ & $209.5 \pm 3.7$ & $212.8 \pm 3.5$ & $421.3 \pm 3.9^{* \# \& a b}$ \\
\hline NC group & $211.6 \pm 3.8$ & $208.5 \pm 3.2$ & $469.2 \pm 4.3^{* a b}$ \\
\hline LncRNA H19-shRNA group & $207.7 \pm 8.3$ & $213.1 \pm 8.6$ & $513.7 \pm 4.8^{\# \text { Sab }}$ \\
\hline
\end{tabular}

Notes: * $P<0.05$ as compared with the normal group; \#, $P<0.05$ as compared with the model group; \&, $P<0.05$ as compared with the empty vector group; $\$, P<0.05$ as compared to the NC group; a, $P<0.05$ as compared with the basal body weight; $\mathrm{b}, P<0.05$ as compared with the body weight after the modeling; lncRNA, long non-coding RNA; NC, negative group; shRNA, short hairpin RNA.

levels in the empty vector, over-expressed lncRNA H19, model, and NC groups increased (all $P<0.05$ ). There was no significant difference in the lncRNA H19-shRNA and normal groups $(P>0.05)$. Compared with the empty vector group, the random blood glucose levels at 12 weeks significantly increased in the over-expressed lncRNA H19 group $(P<0.05)$, while they decreased in the model, $\mathrm{NC}$, and lncRNA H19-shRNA groups compared with the over-expressed lncRNA H19 group, and decreased in the IncRNA H19-shRNA group compared with the model and NC groups $(P<0.05)$. Compared to the basal blood glucose levels, the blood glucose levels after modeling and random blood glucose levels at 12 weeks increased in the empty vector, over-expressed lncRNA H19, model, and NC groups (all $P<0.05$ ). However, there was no significant difference in the basal level of blood glucose in the lncRNA H19-shRNA group $(P>0.05)$. Compared to the blood glucose levels after modeling, the random blood glucose at 12 weeks in the over-expressed lncRNA H19 group, the random blood glucose at 12 weeks significantly increased in the IncRNA H19-shRNA, model and NC groups $(P<0.05)$, but decreased in the lncRNA H19-
shRNA group $(P<0.05)$ (Table 1$)$.

There was no significant difference in body weight before and after modeling in each group (all $P>0.05$ ). As compared with the normal group, the body weight at 12 weeks decreased in the empty vector, over-expressed lncRNA H19, model, and NC groups, but did not change in the lncRNA H19-shRNA group $(P>0.05)$. Compared to the empty vector group, the body weight at 12 weeks was significantly reduced in the over-expressed lncRNA H19 group $(P<0.05)$, and increased in the model, NC and IncRNA H19-shRNA groups when compared with the over-expressed lncRNA H19 group $(P<0.05)$. The body weight at 12 weeks significantly increased in the lncRNA H19-shRNA group compared with the model and NC groups $(P<0.05)$, and there was no statistically significant difference between these two groups $(P>0.05)$. Compared with the basal body weight and the body weight after the modeling, the body weight at 12 weeks significantly decreased in the empty vector, over-expressed lncRNA H19, model and NC groups, while they increased in the lncRNA H19-shRNA group (all $P<0.05$ ) (Table 2). 
Table 3: Positioning navigation and space exploration abilities of rats among six groups by Morris water maze experiment $(n=10)$.

\begin{tabular}{|l|c|c|}
\hline \multicolumn{1}{|c|}{ Group } & Positioning navigation (s) & Space exploration (s) \\
\hline Normal group & $20.10 \pm 7.15$ & $33.13 \pm 5.17$ \\
\hline Model group & $34.25 \pm 11.70^{*}$ & $24.55 \pm 6.34^{*}$ \\
\hline Empty vector group & $35.60 \pm 9.90^{*}$ & $25.24 \pm 5.53^{*}$ \\
\hline Over-expressed lncRNA H19 group & $63.05 \pm 8.30^{* * \&}$ & $10.37 \pm 4.18^{* * \&}$ \\
\hline NC group & $33.68 \pm 11.47^{*}$ & $24.92 \pm 5.17^{*}$ \\
\hline LncRNA H19-shRNA group & $20.70 \pm 7.02^{\$}$ & $33.09 \pm 4.35^{\$}$ \\
\hline
\end{tabular}

Notes: * $P<0.05$ as compared with the normal group; $\#, P<0.05$ as compared with the model group; $\&, P<0.05$ as compared with the empty vector group; $\$, P<0.05$ as compared to the NC group; lncRNA, long non-coding RNA; NC, negative group; shRNA, short hairpin RNA.

A

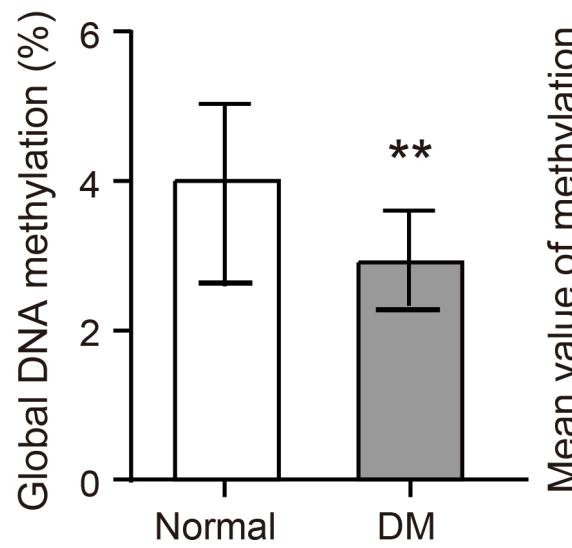

C
B

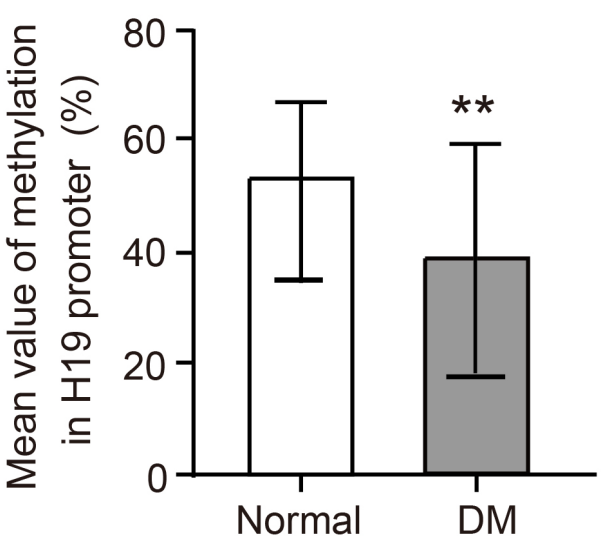

DNA methylation value of IncRNA-H19 promoter
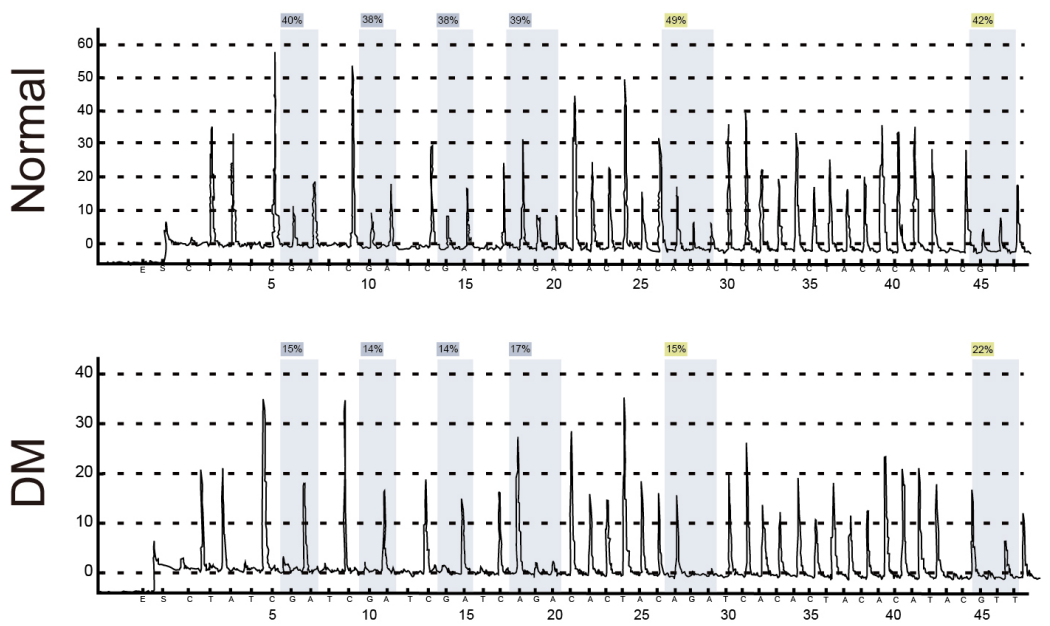

Figure 2: The expression of whole genome methylation in rat model of the hippocampal tissues and the expression of methylation from H19 promoter region of LncRNA H19. Notes: A., Elisa detected the expression of whole genome methylation of the hippocampal tissues; B.,C., the expression of methylation from H19 promoter region of LncRNA H19; $* *$ indicated $P<0.01$ as compared to the normal group; LncRNA = long non-coding RNA. 
Table 4: Primer sequence for qRT-PCR.

\begin{tabular}{|l|c|c|}
\hline \multicolumn{1}{|c|}{ Gene } & Forward primers (5'-3') & Reverse primers (5'-3') \\
\hline LncRNA H19 & TACAACCACTGCACTACCTG & TGGAATGCTTGAAGGCTGCT \\
\hline Wnt3 & GCCTCTGACAAGCCCGAAA & GCGACGCCCCCAATAGTT \\
\hline GSK-3 $\beta$ & GGCTGTGTGTTGGCTGAAT & GATTGCCTCTGGTGGAGTTC \\
\hline$\beta$-catenin & GCTGATTTGATGGAGTTGGA & TCAGCTACTTGTTCTTGAGTGAA \\
\hline TCF-1 & TGGCTTCTACTCCCTGACC & CTGCCTTGGACTCTGCTT \\
\hline Bax & AAGCTGAGCGAGTGTCTCAAG & CAAAGTAGAAAAGGGCGACAAC \\
\hline Bcl-2 & CGCATCAGGAAGGCTAGAGTT & CAGACATTCGGAGACCACACT \\
\hline Caspase-3 & GAGCACTGGAATGTCATCTCGCTCTG & TACAGGAAGTCAGCCTCCACCGGTATC \\
\hline Caspase-8 & TGCCCTCAAGTTCCTGTGCTTGGA & GGATGCTAAGAATG-TCATCTCC \\
\hline GAPDH & ACCACAGTCCATGCCATCAC & TCCACCACCCTGTTGCTGTA \\
\hline
\end{tabular}

Note: qRT-PCR, quantitative real-time polymerace chain reaction.

\section{Positioning navigation and space exploration abilities in $\mathrm{DM}$ rats}

The positioning navigation and space exploration abilities of the rats were studied using the Morris water maze experiments. Compared with the normal group, the latency of positioning navigation significantly increased in the empty vector, over-expressed lncRNA H19, model, and $\mathrm{NC}$ groups, while the duration of space exploration significantly shortened (all $P<0.05$ ). There was no significant difference between the lncRNA H19-shRNA and normal groups $(P>0.05)$. Compared with the model and empty vector groups, the latency of positioning navigation significantly prolonged, while the duration of space exploration significantly shortened in the overexpressed lncRNA H19 group $(P<0.05)$. There was no significant difference between the model and empty vector groups $(P>0.05)$, indicating that the learning and memory abilities of rats had a severe decline in the over-expressed lncRNA H19 group. Compared with the over-expressed IncRNA H19 group, the latency of positioning navigation decreased, and the duration of space exploration in the model, NC, and lncRNA H19-shRNA groups increased ( $P$ $<0.05$ ). Compared with model and NC groups, the latency of positioning navigation in the IncRNA H19-shRNA
A

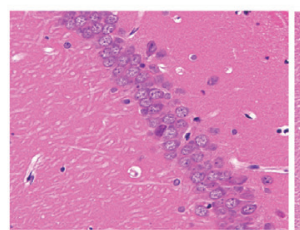

Normal

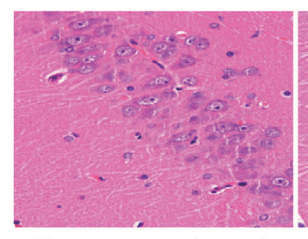

Overexpression of IncRNA H19

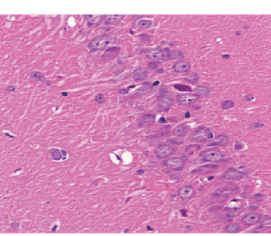

Model

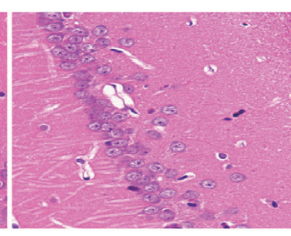

NC

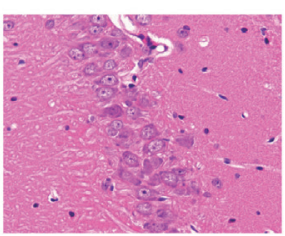

Empty vector

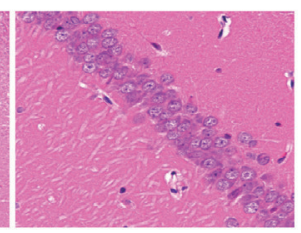

LncRNA H19-shRNA
B

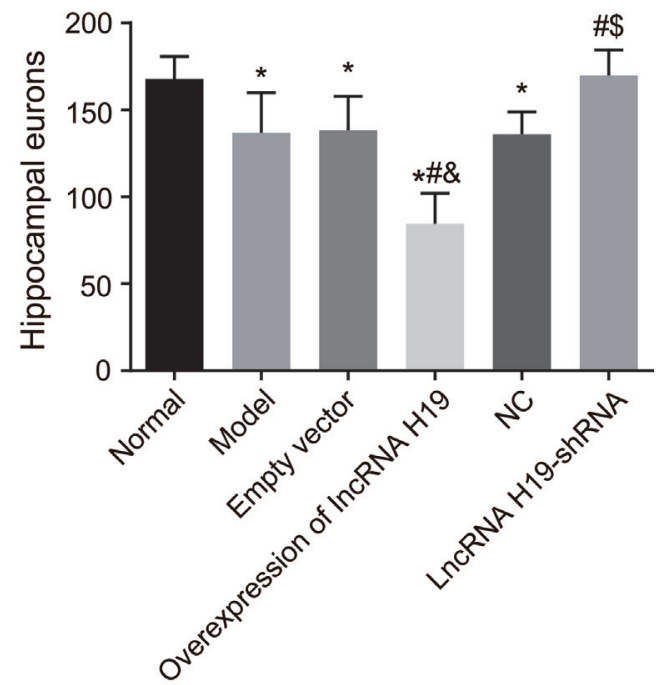

Figure 3: Pathological changes of hippocampal CA1 region of rats in each group and a number of neurons were couted using HE staining $(\times \mathbf{4 0 0})$. Notes: *, $P<0.05$ as compared with the normal group; \#, $P<0.05$ as compared with the model group; \&, $P<$ 0.05 as compared with the empty vector group; $\$, P<0.05$ as compared to the $\mathrm{NC}$ group; $\mathrm{NC}=$ negative control, $\mathrm{HE}=$ Hematoxylin-eosin 
group decreased, while the duration of space exploration increased $(P<0.05)$. There was no significant difference between the model and NC groups $(P>0.05)$. Together, these results suggest that the learning and memory abilities of the rats significantly improve in the lncRNA H19shRNA group (Table 3).

\section{Pathological changes of the hippocampal CA1 region in DM rats}

The neuronal cells in the rat hippocampal CA1 regions were closely arranged; they were round and the nucleus and cytoplasm were clearly visible in the normal group. In the empty vector, over-expressed lncRNA H19, model, and NC groups, the neuronal cells were loosely arranged with changed morphology, including nuclear condensation and cytoplasm reduction, indicating apoptosis. In contrast, cell morphology in the lncRNA H19-shRNA group was similar to the normal group (Figure 3A). In the empty vector, over-expressed lncRNA H19, model and NC groups, the numbers of hippocampal neurons were significantly lower than in the normal group $(P<0.05)$, while there was no significant difference between the normal and IncRNA H19-shRNA groups $(P>0.05)$. Compared with the empty vector group, the numbers of hippocampal neurons in the over-expressed lncRNA H19 group $(P<0.05)$ decreased, suggesting that learning and memory in the over-expressed lncRNA H19 group decreased. On the other hand, learning and memory increased in the model, NC, and lncRNA H19-
shRNA groups when compared with the over-expressed lncRNA H19 group (all $P<0.05$ ). They also increased in the lncRNA H19-shRNA group when compared with the model and NC groups $(P<0.05)$; there was no significant difference between these two groups $(P>0.05)$. Together, these results indicate that learning and memory improved in the rat lncRNA H19-shRNA group (Figure 3B).

\section{Electron microscopy of the hippocampal CA1 region in $\mathrm{DM}$ rats}

The ultrastructural changes of the hippocampal CA1 neurons were mainly manifested as the synaptic changes. Compared with the normal group, the number of synaptosomes in the empty vector group, the overexpressed lncRNA H19 group, the model group and the NC group decreased, while their synaptic degeneration gap increased with a progress of mitochondrial vacuolization inside the synapses. In addition, the depth of condensed matters in synaptosomes was decreased after contacting the synapses. In the IncRNA H19-shRNA group, the ultrastructures were similar to those in the normal group (Figure 4A).

Compared to the normal group, the number of synaptosomes in the empty vector group, the overexpressed lncRNA H19 group, the model group and the NC group decreased $(P<0.05)$, while there was no significant difference between the lncRNA H19-shRNA group and the normal group $(P>0.05)$. Compared with the empty vector group, the number of synaptosomes
A

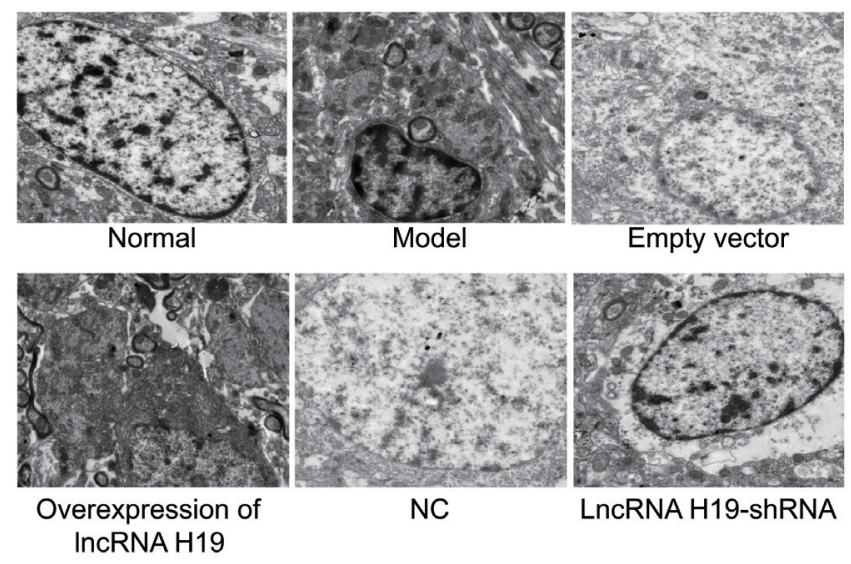

B

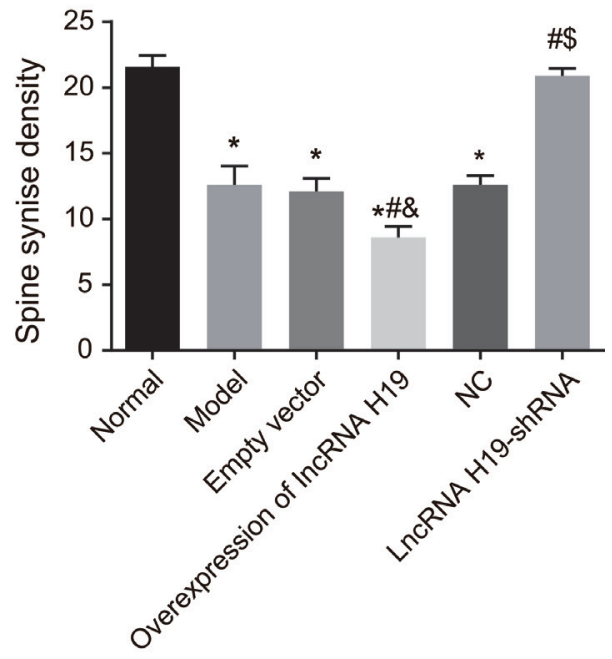

Figure 4: Ultrastructures of hippocampal CA1 region of rats in each group were observed by an electron microscope $(\times \mathbf{4 0 0})$. Notes: A., Electron microscopic images of the ultrastructures in the hippocampal CA1 region of the rats in each group; B., Statistics of synaptosomes in each group of rats; * $P<0.05$ as compared with the normal group; \#, $P<0.05$ as compared with the model group; \&, $P<0.05$ as compared with the empty vector group; $\$, P<0.05$ as compared to the $\mathrm{NC}$ group; $\mathrm{NC}=$ negative control. 
in the over-expressed lncRNA H19 group significantly decreased $(P<0.05)$. Compared with the over-expressed lncRNA H19 group, the number of synaptosomes in the model group, the NC group and the lncRNA H19-shRNA group increased $(P<0.05)$. Compared with the model and the NC groups, the number of synaptosomes in the lncRNA H19-shRNA group increased significantly $(P<$ $0.05)$. There was no significant difference between the model and the NC groups $(P>0.05)$ (Figure 4B).

\section{Wnt signaling protein expression in the hippocampus of DM rats}

Next, we analyzed gene and protein expression of the Wnt signaling pathway proteins in the hippocampus of rats among the six groups. Compared with the normal group, the expressions of Wnt $3, \beta$-catenin and TCF-1 was increased, while the expression of GSK-3 $\beta$ was decreased in the empty vector, over-expressed lncRNA H19, model, and NC groups $(P<0.05)$. There was no significant difference between the lncRNA H19-shRNA and normal groups $(P>0.05)$. In the over-expressed lncRNA H19 group, the expression of Wnt3, $\beta$-catenin, and TCF-1 was up-regulated while the expression of GSK-3 $\beta$ was downregulated when compared to the empty vector group $(P$ $<0.05)$. Compared with the over-expressed lncRNA H19 group, the expressions of Wnt3, $\beta$-catenin, and TCF-1 was reduced and the expression of GSK-3 $\beta$ was increased in the model, NC, and lncRNA H19-shRNA groups ( $P$ $<0.05)$. Compared to the model and NC groups, the expressions of $\mathrm{Wnt} 3, \beta$-catenin and TCF-1 was decreased, and the expression of GSK-3 $\beta$ was increased in the lncRNA H19-shRNA group $(P<0.05)$. There was no significant difference between the model and NC groups (Figure 5).

\section{Apoptosis of hippocampal neurons in DM rats}

We used TUNEL assay to evaluate apoptosis of hippocampal neurons of among the six groups of rats. The nuclei of normal hippocampal neurons were homogeneously blue-stained, and the nuclei of the late apoptotic cells were brown or dark brown. The nuclear pyknosis was irregular, and nuclear fragmentation and nuclear edge condensation could be seen under a high magnification lens. Compared with the normal group, the apoptosis rate of hippocampal neurons was increased in the empty vector, over-expressed lncRNA H19, model, and NC groups $(P<0.05)$, while there was no significant difference between the lncRNA H19-shRNA and normal groups $(P>0.05)$. Compared with the empty vector group, the apoptosis rate of hippocampal neurons was increased in the over-expressed lncRNA H19 group $(P<0.05)$, while it was decreased in the model, NC, and lncRNA H19-shRNA groups. There was no significant difference between the model and NC groups $(P>0.05)$ (Figure 6).
A

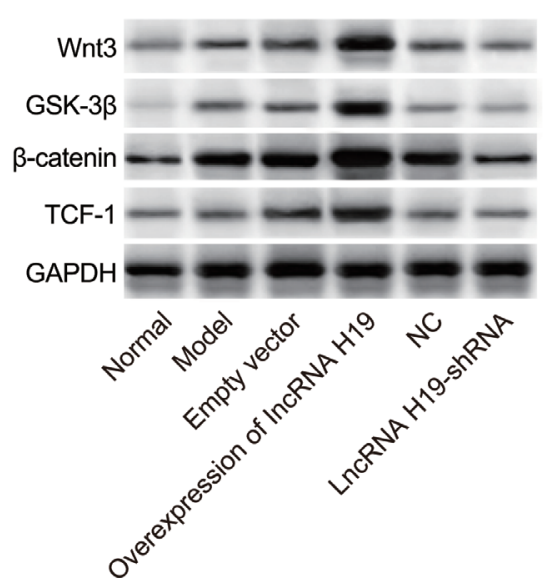

B Normal

- Model

- Empty vector

- Overexpression of IncRNA H19

드 $2.07=\mathrm{NC}$

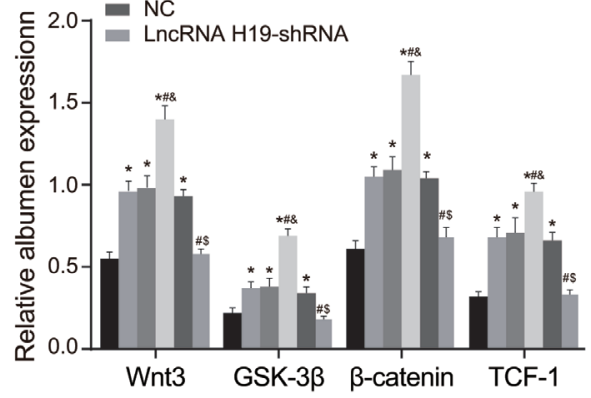

C Normal

Model

- Empty vector

- Overexpression of IncRNA H19

$\left.\stackrel{ }{\frac{}{c}}^{4}\right]=$ LncRNA H19-shRNA

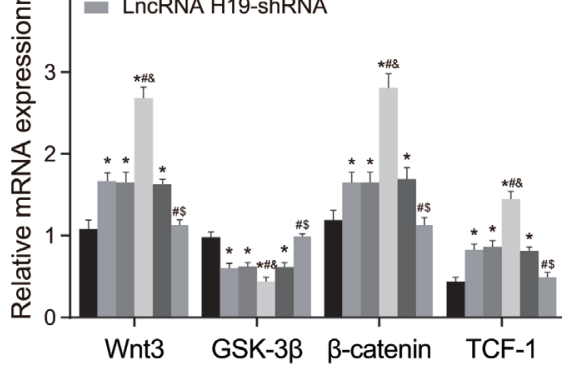

Figure 5: The expressions of Wnt signaling pathway-related proteins in the hippocampus of rats were measured by qRT-PCR and Western blotting. Notes: A., The expressions of Wnt signaling pathway-related proteins in the hippocampus of each group of rats were detected by Western blotting; B., The quantification of the western-blotting; C., The expressions of mRNA of Wnt signaling pathway-related proteins in the hippocampus of rats in each group were detected by qRT-PCR; *, $P<0.05$ as compared with the normal group; \#, $P<0.05$ as compared with the model group; $\&, P<0.05$ as compared with the empty vector group; $\$, P<0.05$ as compared to the $\mathrm{NC}$ group; $\mathrm{NC}=$ negative control; qRT-PCR = quantitative real-time plymerace chain reaction. 


\section{Expression of apoptosis-related proteins in the hippocampus tissues of DM rats}

Compared with the normal group, the protein levels of Bax, caspase- 3 , and caspase- 8 were increased in the empty vector, over-expressed lncRNA H19, model, and NC groups, while the expression of Bcl-2 was decerased $(P<0.05)$. There was no significant difference between the lncRNA H19-shRNA and normal groups $(P>0.05)$.
A

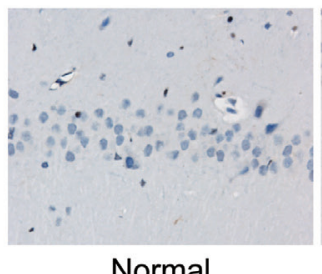

Normal

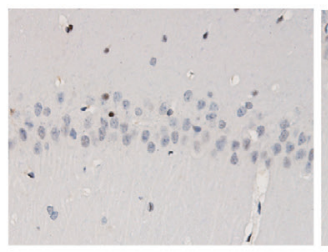

Overexpression of IncRNA H19
B

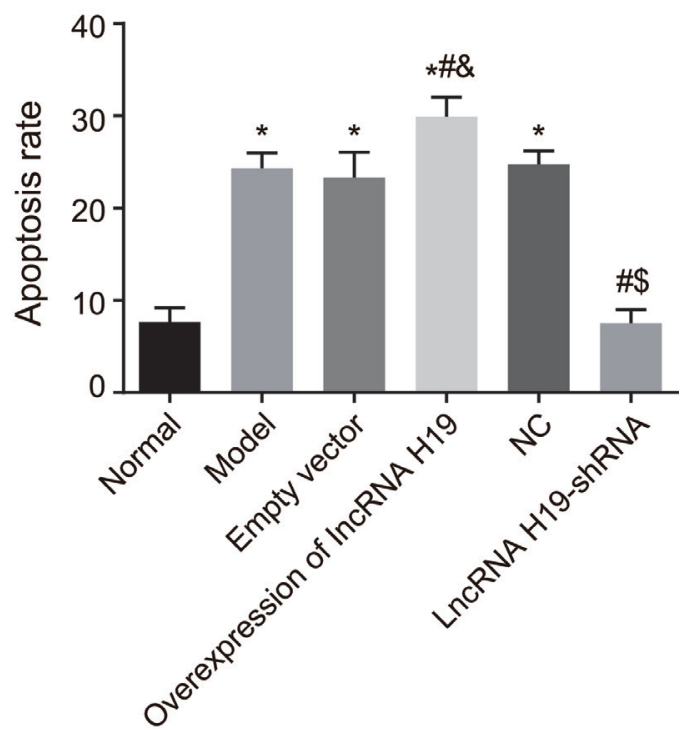

Figure 6: The apoptosis of hippocampal neurons of rats in each group was detected by TUNEL assay $(\times 100)$. Notes: A., The TUNEL staining images of rats in each group; B., The statistics of apoptosis rates; *, $P<0.05$ as compared with the normal group; $\#, P<0.05$ as compared with the model group; \&, $P<0.05$ as compared with the empty vector group; $\$, P<0.05$ as compared to the $\mathrm{NC}$ group; $\mathrm{NC}=$ negative control.

A

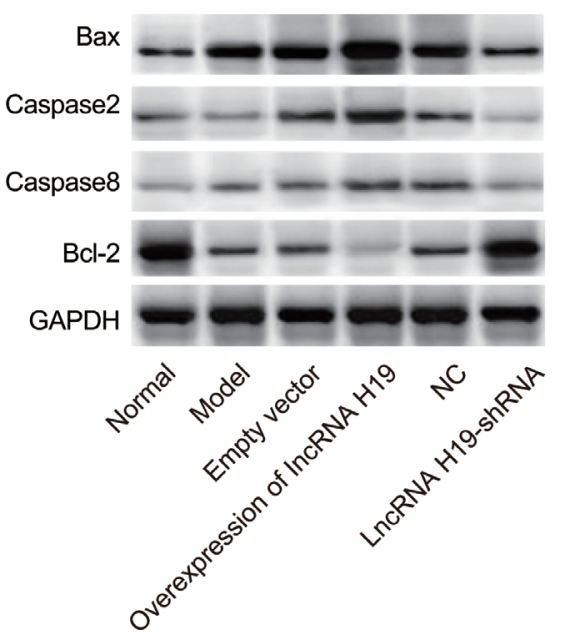

B $\quad$ Normal

- Model

- Empty vector

- Overexpression of IncRNA H19

- NC

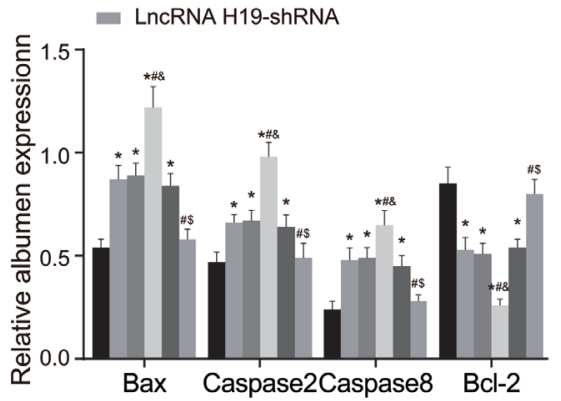
C Normal
Model
- Empty vector
- Overexpression of IncRNA H19

n NC

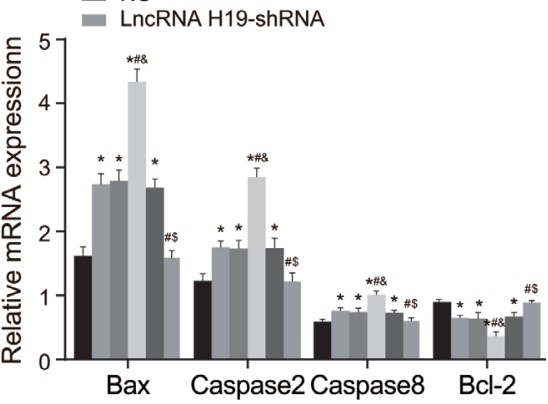

Figure 7: The expressions of apoptosis-related proteins in the hippocampus tissues of rats in each group were detected by qRT-PCR and Western blotting. Notes: A., The expressions of apoptosis-related proteins in the hippocampus tissues of each group of rats were detected by Western blotting; B., The quantification of the western-blotting; C., The expressions of the mRNA of apoptosisrelated proteins in the hippocampus tissues of each group of rats were detected by qRT-PCR; *, $P<0.05$ as compared with the normal group; \#, $P<0.05$ as compared with the model group; \&, $P<0.05$ as compared with the empty vector group; $\$, P<0.05$ as compared to the $\mathrm{NC}$ group; $\mathrm{NC}=$ negative control; qRT-PCR = quantitative real-time plymerace chain reaction. 
Compared to the empty vector group, the expression of Bax, caspase-3, and caspase- 8 was increased, while the expression of Bcl-2 was reduced in the over-expressed lncRNA H19 group $(P<0.05)$. When compared with the over-expressed lncRNA H19 group, the expression of Bax, caspase-3, and caspase- 8 was decreased and the expression of Bcl-2 was increased in the model, $\mathrm{NC}$, and lncRNA H19-shRNA groups $(P<0.05)$. Compared with the model and NC groups, the expression of Bax, caspase- 3 and caspase- 8 was decreased in the lncRNA H19-shRNA group, while the expression of Bcl-2 was upregulated $(P<0.05)$. There was no significant difference between the model and NC groups $(P>0.05)$ (Figure 7).

\section{DISCUSSION}

Defects in hippocampal synaptic plasticity and disorders of memory and learning are the central nervous system complications of DM [15]. As cognitive impairment is mainly attributed to the hippocampal neuron loss [16], understanding the potential impact of lncRNA H19 on the apoptosis of hippocampal neurons via Wnt signaling may provide a theoretical foundation for the development of novel therapeutic strategies against the nervous system complications of DM.

Our present results demonstrate an increased expression of lncRNA H19 in rats with DM. LncRNA $\mathrm{H} 19$ is an imprinted gene at $90 \mathrm{~kb} 3$ ' of IGF2, regulating its expression and imprinting. Abnormal IGF2/H19 methylation has been associated with the development of intrauterine hyperglycemia [17]. In addition, DM induces DNA methylation alterations of H19 in the spermatozoa of rat offsprings [18].

Our study demonstrates a positive correlation between the expression of $\mathrm{Wnt} 3$ and $\beta$-catenin, and lncRNA H19 levels, suggesting that the Wnt signaling is activated by lncRNA H19. As a miRNA sponge, lncRNA H19 can attenuate the endogenous function of miR-22 and miR-141, both of which negatively correlate with the $\beta$-catenin expression, resulting in the activation of $\mathrm{Wnt} / \beta$ catenin signaling [11]. Luo et al have demonstrated that $\mathrm{H} 19$ is associated with zeste homolog 2 (EZH2), and that the association can activate the $\mathrm{Wnt} / \beta$-catenin signaling, thus providing another possible mechanism of the Wnt/ $\beta$-catenin activation by lncRNA H19 [10]. Zhang et al. have confirmed this hypothesis and shown that H19 activates the Wnt signaling [19]. Additionally, the Wnt/ $\beta$ catenin signaling pathway plays a major role in promoting the progression of malignant tumors, indicating that it regulates cell cycle and apoptosis [20]. The Wnt/ $\beta$-catenin signaling activates HT-29-R cells, and H19 knockdown arrests the signaling in the parental cells [21]. Moreover, c-Myc and cyclin D1 are considered as genes of the Wnt/ $\beta$-catenin signaling pathway, and knockdown of CUL4B promotes the cyclin D1- and c-Myc-regulated apoptosis in colorectal cancer cells [20]. In addition, miR-675-5p directly targets H19, and H19 enhances osteogenesis through activating the $\mathrm{Wnt} / \beta$-catenin signaling pathway [11].

Our results demonstrate an increased expression of TCF-1, Bax and caspase 3, but decreased expression of GSK-3 $\beta$ and Bcl-2 in the empty vector, over-expressed lncRNA H19, model, and NC groups compared to the normal group, together with less hippocampal neurons and synaptosomes, and higher apoptosis rate of hippocampal neurons. The Wnt protein signals through a receptor complex, which is comprised of members of the Frizzled and low-density lipoprotein receptor-related protein families, and thus activates a variety of intracellular signaling pathways including the $\beta$-catenin/TCF signaling (known as the canonical Wnt signaling) [22]. Hence, activated Wnt signaling can increase the expression of TCF-1, a member of TCF family [23]. The canonical Wnt signaling has been showed to regulate both cell apoptosis and proliferation in a cell-context dependent manner, by which the activated Wnt/ $\beta$-catenin signaling can not only enhance cell proliferation but also induce apoptosis in various cells [24]. An in vitro study has shown that the activation of $\mathrm{Wnt} / \beta$-catenin signaling can also activate caspase 3 and decrease the mitochondrial membrane potential, resulting in the mitochondria apoptosis [25]. Wnt-induced apoptosis is also found in fibroblasts [26]. Wu et al. have found that activated Wnt signaling promotes macrophage apoptosis and up-regulates Bax and caspase-3, thus verifying our results [27]. The Wnt signaling pathway is activated by the binding of Wnt ligands to a receptor complex composed of a member of the Frizzled family and the low-density lipoproteinreceptor-related protein, which disrupts the regulatory complex of Adenomatous Polyposis Coli APC/Axin/GSK$3 \beta$ by activating cytoplasmic adaptor protein disheveled phosphorylation [27]. In addition, another study has demonstrated that activation of Wnt signaling inactivates GSK-3 $\beta$, leading to dephosphorylation and stabilization of $\beta$-catenin in the cytosol [28]. MiRNAs, such as miR675 , also regulate lncRNAs in glioma [29]. Furthermore, overexpression of IncRNA H19 promotes the proliferation of T/G HA-VSMC in miR-675 in vitro [30].

Finally, prolonged latency of place navigation and the shorter duration of spatial exploration were observed in the over-expressed lncRNA H19, model, and NC groups compared with the normal group, whereas the lncRNA H19-shRNA group exhibited the opposite results. As lncRNA H19 promotes apoptosis of hippocampal neurons and synaptosomes via Wnt signaling, silencing lncRNA H19 could increase the survival of hippocampal neurons. Newborn neurons may be involved in hippocampal functions particularly dependent on the dentate gyrus, such as pattern separation, which may also make distinct contributions to learning and memory [31]. Stranahan et al have conducted a study focused on Morris water maze and shown that spatial memory is impaired in DM rats along 
with decreased hippocampal neurogenesis [3].

Together, our results demonstrate that lncRNA H19 is highly expressed in rats with DM, resulting in activated Wnt signaling and increased apoptosis of hippocampal neurons. However, in rats in the lncRNA H19-shRNA group, apoptosis of hippocampal neurons is effectively suppressed, indicating that inhibition of IncRNA H19 may be a promising method for treatment of cognitive decline of patients with DM. However, further studies are needed to explore how the Wnt signaling can both enhance cell proliferation and induce apoptosis in hippocampal neurons.

\section{MATERIALS AND METHODS}

\section{Ethics statement}

The animal experiments were approved by the ethics committee of the First Hospital of Jilin University. All animal experiments followed the guidelines of the Management and Use of Laboratory Animals published by the National Institutes of Health.

\section{Animal model}

Sixty male SD rats that were 8 weeks old and weighed 180-220 g, were purchased from Shanghai Laboratory Animal Center (SLAC), Shanghai, China. The rats were kept in a clean animal facility with constant humidity and temperature. After one week of adaptation, 50 rats were assigned into a DM group, and the other 10 rats were assigned into a control group with no intervention. The DM rat model was induced by a single injection of a streptozotocin (STZ) solution (SigmaAldrich Chemical Company, St Louis MO, USA) at a dose of $70 \mathrm{mg} / \mathrm{kg}$ of body weight. STZ was dissolved in a sodium citrate buffer, $\mathrm{pH} 4.5$, at $0.1 \mathrm{mmol} / \mathrm{L}$ concentration. One day before the DM induction, the rats fasted for more than $8 \mathrm{~h}$, but water drinking was allowed. After STZ injection, venous blood from rat tails was collected. When the glucose concentration was over $18 \mathrm{mmol} / \mathrm{l}$, the DM model was considered successful, but the rats were not used if their blood glucose levels did not reach the requirement.

\section{Over-expression of IncRNA H19-short hairpin RNA (shRNA) or knock down of the source of IncRNA H19 lentivirus particles}

The plasmid and lentiviral packaging system in the over-expressed lncRNA H19 group and empty vector group were provided from Thermo Scientific Open Biosystems. The high titer lentiviral particles in the over- expressed lncRNA H19 group were constructed, packaged, and concentrated by our laboratory, which was determined by $1 \times 10^{10} \mathrm{TU} / \mathrm{ml}$ final concentration for brain injection. LncRNA H19-shRNA is a GIPZ lentiviral vector carrying the monoclonal small fragment shRNA directed against rats. The negative control group (NC) was a nonsense sequence. Both shRNA plasmids and lentiviral packaging system were purchased from Thermo Scientific Open Biosystems. Prior to the experiment, $8 \%$ glycerol was used as the organic solvent to purify and collect the virus with a titer not less than $1 \times 10^{10} \mathrm{TU} / \mathrm{ml}$, and the final titer for brain injection was $1 \times 10^{10} \mathrm{TU} / \mathrm{ml}$.

\section{Animal grouping}

Animals were divided into 6 groups $(n=10$ in each group): normal group (normal rats), model group (DM rats), empty vector group (DM rats receiving empty vectors), over-expressed lncRNA H19 group (DM rats injected with lncRNA H19 over-expression lentiviral particles), NC group (DM rats injected with nonsense vectors) and lncRNA H19-shRNA group (DM rats injected with lncRNA H19-shRNA lentiviral vectors).

\section{Stereotactic injection}

After successful establishment of the DM model and stabilization of blood glucose, the hippocampal CA1 region was selected as the injection point, and the brain injection was conducted for each animal group. The rats were anesthetized with intra-peritoneal injection of $1 \%$ glutaraldehyde solution at a dose of $40 \mathrm{mg} / \mathrm{kg}$, and their heads were fixed on a stereotaxic apparatus. The injection coordinates were in reference to the rat brain mapping map [32]: $3.5 \sim 4 \mathrm{~mm}$ behind anterior fontanel, incision at \pm 2.8 $\sim 3 \mathrm{~mm}$ beside the midline, and skull dorsal depth of -2.8 $\sim-3.0 \mathrm{~mm}$. In the over-expressed lncRNA H19 group, 5 $\mu \mathrm{l}$ of lncRNA H19 over-expression lentiviral particles at same viral titer were injected at both sides. In the lncRNA H19-shRNA group, $5 \mu \mathrm{l}$ of lncRNA H19-shRNA lentiviral particles [the overall length of H19 (NR_002196), interfere sequence: CGTGACAAGCAGGACATGA] in equal viral titer were injected at both sides. In the $\mathrm{NC}$ group, $5 \mu \mathrm{l}$ of viral particles carrying nonsense sequences were injected at both sides with the same viral titer. Blood glucose and body weight of all rats were monitored during the experiment. Rats in all groups were sacrificed 12 weeks after injection, and whole brain tissues were used for further experiments.

\section{Morris water maze test}

The learning and memory abilities of the rats were evaluated by an animal behavioral learning and memory 
system (Viewpoint Life Sciences, Lyon, France). Before the experiment, adequate amount of water was filled in the water maze, and skim milk powder was used to adjust the light. To minimize errors, experiments were carried out at the same time with the same water temperature. During the first 4 days, positioning navigation experiments were carried out. The rats were placed in water facing the walls, and the rats that climbed onto the platform were observed and recorded by the camera for $1 \mathrm{~min}$. The rats would be manually guided to the platform and stayed there for $30 \mathrm{~s}$, if they still could not climb onto the platform after $1 \mathrm{~min}$, and the latency period was recorded as $1 \mathrm{~min}$. The learning ability of rats in each group was mainly manifested as the latency period in the first 4 days. Space exploration experiments were carried out in the fifth day: the platform was withdrawn, and the rats were placed into the water from the B quadrant. The results of the memory capacity was the swimming time in the D quadrant during a 1-min period.

\section{Hematoxylin-eosin (HE) staining and cell counting}

After blood collection, whole brain tissues were quickly removed and placed on a glacial table, washed with saline, fixed in $4 \%$ formaldehyde solution, embedded with paraffin, and sliced. The tissues were then stained with $\mathrm{HE}$ for $5 \mathrm{~min}$, differentiated, washed, stained again with HE, dehydrated with gradient alcohol, and mounted with a neutral gum in order to be observed under an inverted microscope. The cell count of hippocampal neuron was centered at the junction of the hippocampal CA1 region and the extension line from the end of the upper and lower branches in the dentate gyrus of the hippocampal tissue. Centered at this junction, and extended to both directions by $0.5 \mathrm{~mm}$, a $1 \mathrm{~mm}$ length was chosen as the experimental count range for the cells in the hippocampal CA1 region. The cells were counted and photographed.

\section{Electron microscopy}

After blood collection, whole brain tissues were placed on a glacial table, washed with saline, and fixed with $2.5 \%$ glutaraldehyde solution. The hippocampus tissues were dissected under an inverted microscope. Tissue sections of $1 \mathrm{~mm}^{3}$ in the hippocampal CA1 region were fixed with $2.5 \%$ glutaraldehyde solution, washed with phosphate buffered saline (PBS), dehydrated, embedded, sliced, and stained with lead citrate and acetic acid. The ultrastructure of synaptosomes and surrounding organelles in hippocampal CA1 region was observed under an electron microscope. The number of synaptosomes was counted and analyzed by the Physical Disector (Disector Countor 1.0) software.

\section{Western blotting}

Tissue proteins were extracted and their concentration was determined using a BCA kit (Wuhan Boster Biological Technology Ltd., Wuhan, Hubei, China). The protein extracts were boiled in a sample buffer for $10 \mathrm{~min}$, separated on a $10 \%$ polyacrylamide gel, and transferred to PVDF membrane. The membranes were blocked with 5\% bovine serum albumin ( $1 \mathrm{~h}, \mathrm{RT})$, and incubated with primary antibodies (Cell Signaling Technology) for Wnt3 (1:1000 dilution, \#9248), GSK$3 \beta$ (1:1000 dilution, \#8240), glyceraldehyde phosphate dehydrogenase (GAPDH) (1:500 dilution, \#5174), and $\beta$-catenin (1:1000 dilution, \#9566). In addition, antibodies against TCF-1 (1:1000 dilution, sc-79542), Bax (1:1000 dilution, sc-23959), Bcl-2 (1:1000 dilution, sc-7382), caspase-3 (1:1000 dilution, sc-65496), and caspase-8 (1:1000 dilution, sc-81656) purchased from Santa Cruz Biotechnology were used. The membranes were then washed in TBST and incubated with corresponding secondary antibodies (1 h, RT). GAPDH was used as an internal reference. Bio-Rad Gel DOC EZ imager was used to develop the membranes. The images were evaluated using the Image J software.

\section{Quantitative real-time polymerase chain reaction (qRT-PCR)}

Total RNA was extracted and reverse transcription of cDNA were performed according to the kit instructions (QIAGEN, Valencia, CA). Primers were designed using the Primer 5.0 primer design software according to the gene sequences published in the Genbank database. The primers (Table 4) were synthesized by Shanghai Sangon Biological Engineering Technology \& Services Co., Ltd. (Shanghai, China). The reaction system of PCR was 20 $\mu \mathrm{l}: 10 \mu \mathrm{l}$ of SYBR PremixExTaq, $0.8 \mu \mathrm{l}$ of forward and reverse primers, $0.4 \mu \mathrm{l}$ of ROX Reference Dye II, $2 \mu \mathrm{l}$ of DNA template, and $6.0 \mu \mathrm{l}$ of $\mathrm{dH}_{2} \mathrm{O}$. The reaction conditions were as follows: pre-denaturation at $95^{\circ} \mathrm{C}$ for $30 \mathrm{~s}, 40$ cycles of denaturation at $95^{\circ} \mathrm{C}$ for $5 \mathrm{~s}$, annealing at $60^{\circ} \mathrm{C}$ for $30 \mathrm{~s}$, and extension at $72^{\circ} \mathrm{C}$ for $30 \mathrm{~s}$. The internal control used in our study was GAPDH. The relative expression of the target gene was calculated as $2^{-\Delta \Delta} \mathrm{Ct}$, wherein $\mathrm{Ct}$ value is the cycle threshold [33].

\section{TUNEL staining}

TUNEL detection kit (F. Hoffmann-La Roche Ltd., Basel, Switzerland) was used to detect the apoptosis. The hippocampus tissues were fixed for $30 \mathrm{~min}$ at room temperature, rinsed with $\mathrm{PBS}$, and immersed in $0.2 \%$ Triton X-100 solution for $5 \mathrm{~min}$ to enhance the permeability. Subsequently, the samples were washed 
with PBS and dried, and $50 \mu$ of reaction mixture (ratio of concentrated enzyme solution and labeling solution was 1: 9) were added. The samples were washed with PBS and observed under a fluorescence microscope. In each slide, 5 view fields were randomly selected and the number of apoptotic cells was counted as the apoptotic rate $=$ the number of apoptotic cells / total cells $\times 100 \%$.

\section{Whole genome methylation and detection of H19 promoter region}

DNA from the hippocampal tissues was extracted, and methylation was analyzed using the whole gene methylation kit.

The pyrophosphate sequencing was used to detect DNA methylation in DMR region two locus of Lnc RNA H19. The primers used for sequencing were synthesized (Qiagen, Hilden, Germany). DNA from $100 \mathrm{ng}$ pyrophosphate was amplified using a reaction system that included $5 \times$ PCR buffer, $10 \mathrm{mM}$ dNTP, $50 \mathrm{mM}$ upstream and downstream primers, and $1 \mathrm{U}$ DNA polymerase. The PCR conditions were: $95^{\circ} \mathrm{C}$ for $3 \mathrm{~min}$, cycled 40 times at $95^{\circ} \mathrm{C}$ for $30 \mathrm{~s}$, annealed at $60^{\circ} \mathrm{C}$ for $30 \mathrm{~s}$ and $72^{\circ} \mathrm{C}$ for $1 \mathrm{~min}$, and finally at $72^{\circ} \mathrm{C}$ for $7 \mathrm{~min}$. The sequencing program of PCR product was tested using Pyromark Q96 ID. The methylation was analyzed using the Allele quantitative software. All samples was measured 3 times.

\section{Statistical analysis}

Data were analyzed using the SPSS18.0 statistical software, and the results were expressed as mean $\pm \mathrm{s}$. The comparisons between two groups with normal distributions were detected using the $t$ test. The comparisons among multiple groups were detected by One-Way analysis of variance (ANOVA), and $P<0.05$ indicated a statistically significant difference.

\section{CONFLICTS OF INTEREST}

No potential conflicts of interest were disclosed.

\section{REFERENCES}

1. American Diabetes Association. Diagnosis and classification of diabetes mellitus. Diabetes Care. 2012; 35 Suppl 1: S64-71. doi: 10.2337/dc12-s064.

2. Farrag W, Eid M. Association of the $\mathrm{C} 242 \mathrm{~T}$ polymorphism of the p22 phox gene with advanced carotid atherosclerosis in type 2 diabetes. Mol Med Rep. 2008; 1: 679-84. doi: 10.3892/mmr_00000012.

3. Stranahan AM, Arumugam TV, Cutler RG, Lee K, Egan JM, Mattson MP. Diabetes impairs hippocampal function through glucocorticoid-mediated effects on new and mature neurons. Nat Neurosci. 2008; 11: 309-17. doi: 10.1038/ nn2055.

4. Stranahan AM, Norman ED, Lee K, Cutler RG, Telljohann RS, Egan JM, Mattson MP. Diet-induced insulin resistance impairs hippocampal synaptic plasticity and cognition in middle-aged rats. Hippocampus. 2008; 18: 1085-8. doi: 10.1002/hipo.20470.

5. Ho N, Sommers MS, Lucki I. Effects of diabetes on hippocampal neurogenesis: links to cognition and depression. Neurosci Biobehav Rev. 2013; 37: 1346-62. doi: 10.1016/j.neubiorev.2013.03.010.

6. Barry G. Integrating the roles of long and small non-coding RNA in brain function and disease. Mol Psychiatry. 2014; 19: 410-6. doi: 10.1038/mp.2013.196.

7. Yang F, Bi J, Xue X, Zheng L, Zhi K, Hua J, Fang G. Up-regulated long non-coding RNA H19 contributes to proliferation of gastric cancer cells. FEBS J. 2012; 279: 3159-65. doi: 10.1111/j.1742-4658.2012.08694.x.

8. Shi Y, Wang Y, Luan W, Wang P, Tao T, Zhang J, Qian J, Liu N, You Y. Long non-coding RNA H19 promotes glioma cell invasion by deriving miR-675. PLoS One. 2014; 9: e86295. doi: 10.1371/journal.pone.0086295.

9. Yang C, Tang R, Ma X, Wang Y, Luo D, Xu Z, Zhu Y, Yang L. Tag SNPs in long non-coding RNA H19 contribute to susceptibility to gastric cancer in the Chinese Han population. Oncotarget. 2015; 6: 15311-20. doi: 10.18632/ oncotarget. 3840 .

10. Luo M, Li Z, Wang W, Zeng Y, Liu Z, Qiu J. Long noncoding RNA H19 increases bladder cancer metastasis by associating with EZH2 and inhibiting E-cadherin expression. Cancer Lett. 2013; 333: 213-21. doi: 10.1016/j. canlet.2013.01.033.

11. Liang WC, Fu WM, Wang YB, Sun YX, Xu LL, Wong CW, Chan KM, Li G, Waye MM, Zhang JF. H19 activates Wnt signaling and promotes osteoblast differentiation by functioning as a competing endogenous RNA. Sci Rep. 2016; 6: 20121. doi: 10.1038/srep20121.

12. Lie DC, Colamarino SA, Song HJ, Desire L, Mira H, Consiglio A, Lein ES, Jessberger S, Lansford H, Dearie AR, Gage FH. Wnt signalling regulates adult hippocampal neurogenesis. Nature. 2005; 437: 1370-5. doi: 10.1038/ nature 04108 .

13. Machon O, Backman M, Machonova O, Kozmik Z, Vacik T, Andersen L, Krauss S. A dynamic gradient of Wnt signaling controls initiation of neurogenesis in the mammalian cortex and cellular specification in the hippocampus. Dev Biol. 2007; 311: 223-37. doi: 10.1016/j. ydbio.2007.08.038.

14. Simon M, Grandage VL, Linch DC, Khwaja A. Constitutive activation of the Wnt/beta-catenin signalling pathway in acute myeloid leukaemia. Oncogene. 2005; 24: 2410-20. doi: 10.1038/sj.onc. 1208431.

15. Kamal A, Biessels GJ, Gispen WH, Ramakers GM. Synaptic transmission changes in the pyramidal cells of the 
hippocampus in streptozotocin-induced diabetes mellitus in rats. Brain Res. 2006; 1073-1074: 276-80. doi: 10.1016/j. brainres.2005.12.070.

16. Sima AA, Li ZG. The effect of C-peptide on cognitive dysfunction and hippocampal apoptosis in type 1 diabetic rats. Diabetes. 2005; 54: 1497-505.

17. Ding GL, Wang FF, Shu J, Tian S, Jiang Y, Zhang D, Wang N, Luo Q, Zhang Y, Jin F, Leung PC, Sheng JZ, Huang HF. Transgenerational glucose intolerance with Igf2/H19 epigenetic alterations in mouse islet induced by intrauterine hyperglycemia. Diabetes. 2012; 61: 1133-42. doi: 10.2337/ db11-1314.

18. Ge ZJ, Liang QX, Hou Y, Han ZM, Schatten H, Sun QY, Zhang CL. Maternal obesity and diabetes may cause DNA methylation alteration in the spermatozoa of offspring in mice. Reprod Biol Endocrinol. 2014; 12: 29. doi: 10.1186/1477-7827-12-29.

19. Zhang Q, Su M, Lu G, Wang J. The complexity of bladder cancer: long noncoding RNAs are on the stage. Mol Cancer. 2013; 12: 101. doi: 10.1186/1476-4598-12-101.

20. Song B, Zhan H, Bian Q, Li J. Knockdown of CUL4B inhibits proliferation and promotes apoptosis of colorectal cancer cells through suppressing the Wnt/beta-catenin signaling pathway. Int J Clin Exp Pathol. 2015; 8: $10394-$ 402.

21. Wu KF, Liang WC, Feng L, Pang JX, Waye MM, Zhang JF, Fu WM. H19 mediates methotrexate resistance in colorectal cancer through activating Wnt/beta-catenin pathway. Exp Cell Res. 2017; 350: 312-7. doi: 10.1016/j. yexcr.2016.12.003.

22. Hirabayashi Y, Itoh Y, Tabata H, Nakajima K, Akiyama T, Masuyama N, Gotoh Y. The Wnt/beta-catenin pathway directs neuronal differentiation of cortical neural precursor cells. Development. 2004; 131: 2791-801. doi: 10.1242/ dev.01165.

23. Herzig M, Savarese F, Novatchkova M, Semb H, Christofori G. Tumor progression induced by the loss of E-cadherin independent of beta-catenin/Tcf-mediated Wnt signaling. Oncogene. 2007; 26: 2290-8. doi: 10.1038/sj.onc.1210029.

24. Zimmerman ZF, Kulikauskas RM, Bomsztyk K, Moon RT, Chien AJ. Activation of Wnt/beta-catenin signaling increases apoptosis in melanoma cells treated with trail. PLoS One. 2013; 8: e69593. doi: 10.1371/journal. pone.0069593.

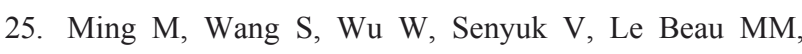
Nucifora G, Qian Z. Activation of Wnt/beta-catenin protein signaling induces mitochondria-mediated apoptosis in hematopoietic progenitor cells. J Biol Chem. 2012; 287: 22683-90. doi: 10.1074/jbc.M112.342089.

26. Kim K, Pang KM, Evans M, Hay ED. Overexpression of beta-catenin induces apoptosis independent of its transactivation function with LEF-1 or the involvement of major G1 cell cycle regulators. Mol Biol Cell. 2000; 11: 3509-23.

27. Wu X, Deng G, Hao X, Li Y, Zeng J, Ma C, He Y, Liu $\mathrm{X}$, Wang Y. A caspase-dependent pathway is involved in $\mathrm{Wnt} /$ beta-catenin signaling promoted apoptosis in Bacillus Calmette-Guerin infected RAW264.7 macrophages. Int J Mol Sci. 2014; 15: 5045-62. doi: 10.3390/ijms15035045.

28. Ji XK, Xie YK, Zhong JQ, Xu QG, Zeng QQ, Wang Y, Zhang QY, Shan YF. GSK-3beta suppresses the proliferation of rat hepatic oval cells through modulating Wnt/beta-catenin signaling pathway. Acta Pharmacol Sin. 2015; 36: 334-42. doi: 10.1038/aps.2014.150.

29. Shi J, Dong B, Cao J, Mao Y, Guan W, Peng Y, Wang S. Long non-coding RNA in glioma: signaling pathways. Oncotarget. 2017; 8:27582-27592. doi: 10.18632/ oncotarget.15175.

30. Lv J, Wang L, Zhang J, Lin R, Wang L, Sun W, Wu H, Xin S. Long noncoding RNA H19-derived miR-675 aggravates restenosis by targeting PTEN. Biochem Biophys Res Commun. 2017. doi: 10.1016/j.bbrc.2017.01.011.

31. Deng W, Aimone JB, Gage FH. New neurons and new memories: how does adult hippocampal neurogenesis affect learning and memory? Nat Rev Neurosci. 2010; 11: 339-50. doi: $10.1038 / \mathrm{nrn} 2822$.

32. Paxinos G WC. The rat brain in stereotaxic coordinates. 5th ed San Diego:Elsevier Academic Press. 2007.

33. Smith DL, Comer TP. Gastrocolic fistula, a rare complication of benign gastric ulcer: report of a case. Dis Colon Rectum. 1974; 17: 769-70. 